\title{
An Overview of Arachnoid Webs
}

\section{Rohit Aiyer $^{1 *}$, Lynne Voutsinas ${ }^{2}$, Yasir El-Sherif}

'Department of Psychiatry, Hofstra Northwell Health - Staten Island University Hospital, USA ${ }^{2}$ Department of Radiology, Hofstra Northwell Health - Staten Island University Hospital, USA

${ }^{3}$ Department of Neurology, Hofstra Northwell Health - Staten Island University Hospital, USA

\section{Article Info}

\section{Article Notes}

Received: August 25, 2016

Accepted: September 20, 2016

\section{*Correspondence:}

Dr. Rohit Aiyer, M.D

Hofstra Northwell Health - Staten Island University Hospital, 475 Seaview Avenue

Staten Island, New York, USA, Telephone: 718-226-6973; E-mail: raiyer@northwell.edu

(c) 2016 Aiyer R. This article is distributed under the terms of the Creative Commons Attribution 4.0 International License
Arachnoid webs are abnormal formations of arachnoid membrane in the spinal subarachnoid space that prevents movement of cerebrospinal fluid (CSF) in the longitudinal direction ${ }^{1}$.These webs represent intradural extramedullary transverse bands of arachnoid tissue that extend to the dorsal surface of the spinal cord resulting in dorsal indentation ${ }^{2}$. These arachnoid webs may represent a variant of an arachnoid cyst ${ }^{3}$. However, they may also represent localized arachnoiditis ${ }^{3}$.

To identify arachnoid webs that cause idiopathic syringomyelia, an important marker is the abrupt termination of a syrinx cavity and a small ventral displacement of the spinal cord next to the termination of the syrinx cavity ${ }^{4}$. There are many causes of syringomyelia, and the formation of an arachnoid web is one the etiologies. Other causes of syringomyelia include: idiopathic, primary, post-traumatic, associated with non-traumatic arachnoid pathologies and associated with adhesive arachnoiditis ${ }^{1}$. At present, it is unknown how the non-traumatic syringomyelias are formed with regards to whether they are congenital or acquired ${ }^{1}$. Anterior spinal cord herniation is a rare disorder that does not have a clearly identified pathogenesis in a majority of cases. Various mechanisms have been speculated to explain and understand the initial dural defect as well as subsequent cord herniation. The majority of patients $(80 \%)$ with spinal cord herniations occur in the thoracic spine, predominantly at the third to seventh thoracic vertebra (T3T7) levels. In cord herniation, the cord tissue protrudes through a ventral dural defect, and the presence of a focal deformity along the ventral part of the cord is usually present. Also, when the ventral spinal cord is closely opposed to the anterior dura, it may cause difficulty differentiating between a dorsal arachnoid web and an anterior spinal cord herniation ${ }^{5}$. On the other hand, arachnoid cysts are identified by the presence of smooth wide scalloping of the cord surface, and that the structures fill slower than the other parts of the subarachnoid space ${ }^{6}$. A characteristic finding on MRI for spinal cord herniation and intradural arachnoid cysts are focal anterior displacements of the spinal cord with enlargement of the dorsal subarachnoid space ${ }^{7}$. Reardon et al described the presence of an arachnoid web in the dorsal subarachnoid space that caused focal indentation of the upper thoracic spinal cord without an intradural mass or spinal cord herniation ${ }^{5}$. They reported 14 patients who showed focal dorsal indentation in the upper thoracic spinal cord, and seven of the patients underwent surgery following imaging studies $^{5}$. 
Diagnosis is made by neuroimaging, most often on sagittal magnetic resonance imaging (MRI) and computed tomography (CT) myelographic images. A classic MRI and CT myelogram appearance of an arachnoid web is the "scalpel sign", due to the close resemblance on sagittal imaging to a scalpel with its blade pointing posteriorly ${ }^{5}$. This "scalpel sign" is evident in figures 1 and $2^{8}$. If noted on imaging, the differential diagnosis of the "scalpel sign" includes dorsal arachnoid web, ventral spinal cord herniation and dorsal arachnoid cyst. Imaging is essential

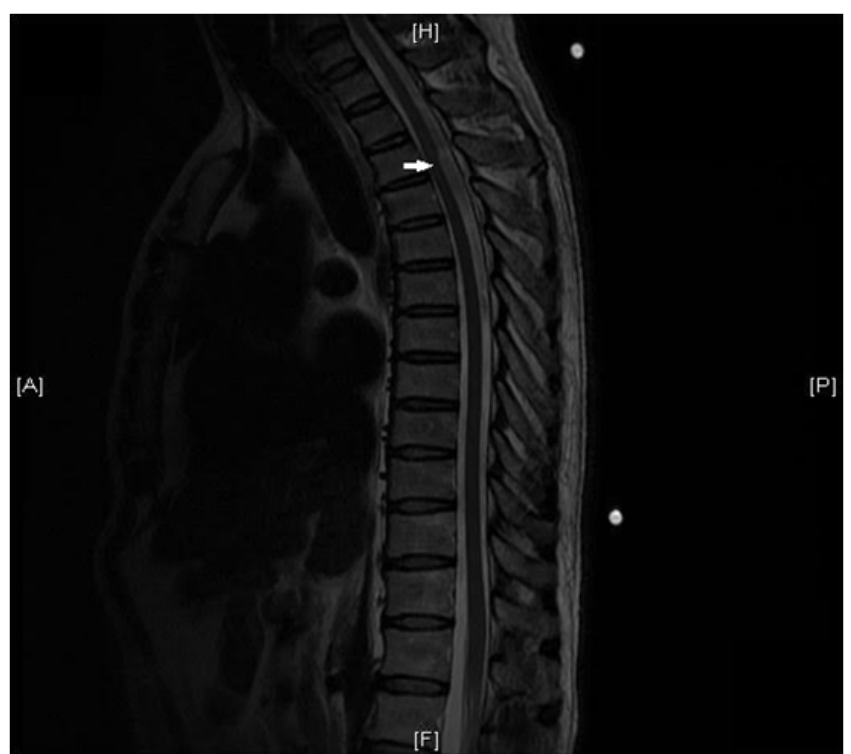

Figure 1: MRI Thoracic Spine - Sagittal T2-weighted sequence. At T3-T4 the arrow highlights the focal distortion of the posterior aspect of the spinal cord with the spinal cord displaced anteriorly and widening of the dorsal cerebrospinal fluid (CSF) space.

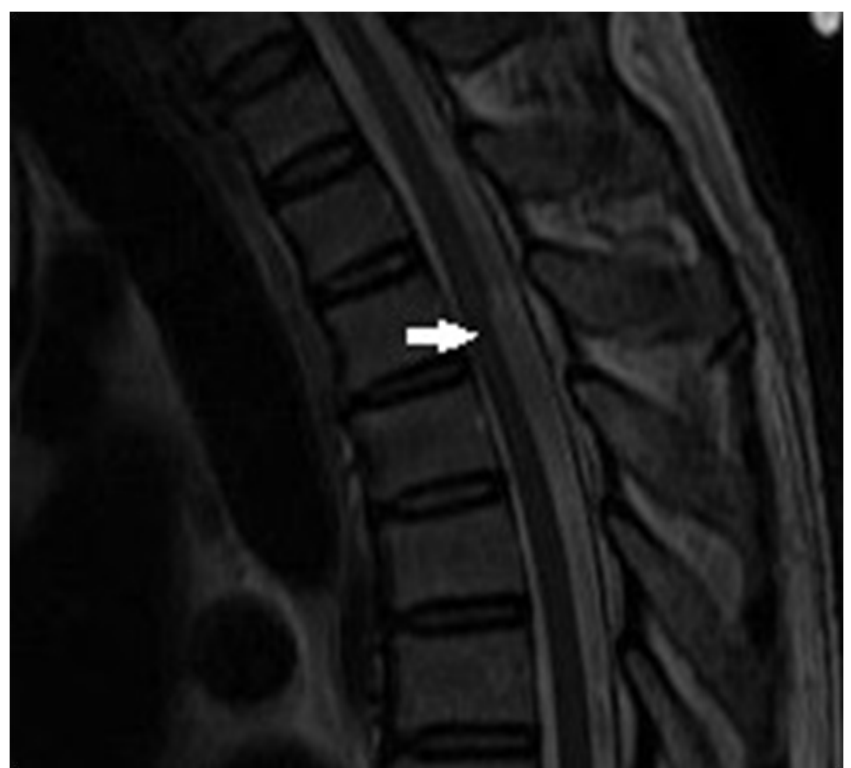

Figure 2: MRI Thoracic Spine - Sagittal T2-weighted sequence. A close up of the dorsal thoracic arachnoid web. as it can help a clinician differentiate herniation from an arachnoid cyst or web ${ }^{5}$. For instance, with cord herniation, the cord tissue protrudes through a ventral dural defect, with the focal deformity located along the ventral aspect of the spinal cord ${ }^{7}$. MRI technique is susceptible to movement artifacts, and arachnoid webs can be missed even on MRI CSF flow sequences. This is why CT myelography remains to be the definitive, preferred imaging method ${ }^{9}$. Arachnoid webs are not easily seen with conventional MR imaging or CT myelography due to their relatively thin width compared to other anatomical structures ${ }^{10}$. A large study of 125 patients with idiopathic syringomyelia was conducted by Mauer et al and investigated the use of cardiac gated phase contrast MRI of CSF flow in the spine ${ }^{11}$. There is conclusive evidence that this technique was superior to CT myelography for arachnoid web imaging ${ }^{11}$. In addition, this mode of imaging in multiple axial planes has been used to quantitatively analyze CSF flow in those particular planes for diagnosis of arachnoid webs ${ }^{10}$.

Spinal arachnoid webs are relatively under reported, with only 31 documented cases in literature ${ }^{8}$. A review of many case reports and the systematic reviews by Groen et $a l^{12}$ and Imagama et $a l^{13}$ provide evidence that even patients with long-standing neurologic symptoms due to arachnoid webs can experience significant recovery after treatment is commenced. The primary treatment goals are to resolve or improve the patient's neurologic symptoms and avoid recurrence of spinal cord herniation or incarceration. Consequently, treatment involves surgical reduction and release of the spinal cord, followed by surgical widening of the dural defect or closure of the defect with an anterior patch or suture. Other surgical techniques include placement of a fat graft, repair of a bone defect and placement of a posterior dural patch to manage the dorsal arachnoid web ${ }^{11}$.

Co-morbidities with arachnoid webs are an occurrence. As mentioned earlier, there appears to be a relationship between the presence of a dorsal arachnoid web and syringomyelia. A case report published by Sridharan and Heilman suggested that the determining factor for syringomyelia formation above or below the level of an arachnoid web is related to the CSF pressure differential on either side of the web ${ }^{4}$. As a result, a greater CSF pressure rostrally would result in syringomyelia formation caudal to the web and vice versa. This is noteworthy since a lower CSF pressure on one side of a subarachnoid obstruction leads to cord distension causing the CSF to enter the cord via small perivascular spaces ${ }^{7}$.

If the lesion is a simple arachnoid web, it is essential to accurately and efficiently quantify CSF flow and illustrate the location of the arachnoid web ${ }^{1}$. The thin structure of the arachnoid web is a reason why routine MRI scans may overlook or miss an arachnoid web diagnosis. Furthermore, 
the topological relationship of the arachnoid web to the syrinx is inconsistent, causing for more difficulty in identifying on imaging ${ }^{1}$. Previous literature indicates that CT myelography is the preferred investigation to report arachnoid webs ${ }^{7}$. However, newer studies now support cardiac gated MRI techniques as possibly a preferential imaging choice for identifying CSF flow, compared to CT myelography ${ }^{7}$. This was specifically shown in 125 patients in a study by Mauer et $a l^{11}$. Nevertheless, MRI images are vulnerable to movement artifacts, and therefore arachnoid webs can be missed ${ }^{7}$.

Treating arachnoid webs include a variety of options: lysis of the web, duraplasty and syringosubarachnoid and syringopleural shunts ${ }^{14,15}$. One study illustrated the benefits of shunts with arachnoid web treatment, however, it is still not a preferred choice due to the high complication rate, which include formation of further arachnoid adhesions as well as tethering ${ }^{16}$. Lysing of the web is however more difficult, from a technical point of view. Studies indicate that shunts only help in $11 \%$ of patients while lysis generates a slightly higher success rate of $31 \%$. Complication rates for arachnolysis and shunt insertion was $17 \%$ and $18 \%$, respectively ${ }^{16}$.

In conclusion, it is evident that arachnoid webs are rare in the clinical world, but should be considered when patients are presenting with concerning, suggestive neurologic symptoms. Imaging is critical for clinicians to make a quick and accurate diagnosis, which would also allow for early surgical intervention.

\section{References}

1. Chang HS, Nagai A, Oya S, Matsui T. Dorsal spinal arachnoid web diagnosed with the quantitative measurement of cerebrospinal fluid flow on magnetic resonance imaging: report of 2 cases. Journal of Neurosurgery: Spine. 2014; 20(2): 227-233.

2. Paramore CG. Dorsal arachnoid web with spinal cord compression: variant of an arachnoid cyst? Report of two cases. Journal of Neurosurgery. 2000; 93:287-90.

3. Brodbelt AR, Stoodley MA. Case Report Syringomyelia and the arachnoid web. Acta Neurochirugica. 2003; 145: 707-711.
4. Sridharan A, Heilman CB. Transverse dorsal arachnoid web and syringomyelia: case report. Neurosurgery. 2009; 65 (1): E216-7.

5. Reardon MA, Raghavan P, Carpenter-Bailey K, Mukherjee S, Smith JS, Matsumoto JA, Yen CP, Shaffrey ME, Lee RR, Shaffrey CI, Wintermark M. Dorsal Thoracic Arachnoid Web and the "Scalpel Sign": A Distinct Clinical-Radiology Entity. American Journal of Neuroradiology. 2013; 34 (5): 1104-10.

6. Haber MD, Nguyen DD, Li S. Differentiation of Idiopathic Spinal Cord Herniation from CSF-isointense intraspinal extra-medullary lesions displacing the cord. Radiographics. 2014; 34(2): 313-329.

7. Lee JY, Lee JW, Lee GY, Kang HS. Focal Anterior Displacement of the Thoracic Spinal Cord without Evidence of Spinal Cord Herniation or an Intradural Mass. Korean Journal of Radiology. 2014; 15(6): 733738.

8. Aiyer R, El-Sherif Y, Voutsinas L. Dorsal thoracic arachnoid web presenting as neuropathic pain: 'Scalpel' sign found on MRI. The Neuroradiology Journal. June 17, 2015.

9. Saval PP, Zafar A, Carroll TA. Syringomyelia secondary to "occult" dorsal arachnoid webs: report of two cases with review of literature. Journal of Craniovertebral Junction \& Spine. 2016; 7(2): 101-4.

10. Chang H.S., Nagai A., Oya S., and Matsui T.: Dorsal spinal arachnoid web diagnosed with the quantitative measurement of cerebrospinal fluid flow on magnetic resonance imaging. Journal of Neurosurgery Spine. 2014; 20: 227-233.

11. Mauer UM, Freude G, Danz B, Kunz U. Cardiac-gated phase-contrast magnetic resonance imaging of cerebrospinal fluid flow in the diagnosis of idiopathic syringomyelia. Neurosurgery. 2008; 3:113944.

12. Groen RJ, Middel B, Meilof JF. Operative treatment of anterior thoracic spinal cord herniation: three new cases and an individual patient data meta-analysis of 126 case reports. Neurosurgery. 2009; 64(3): 145160.

13. Imagama S, Matsuyama Y, Sakai Y. Image classification of idiopathic spinal cord herniation based on symptom severity and surgical outcome: a multicenter study. Journal of Neurosurgery and Spine. 2009;11(3):310-319.

14. Batzdorf U, Klekamp J, Johnson JP. A critical appraisal of syrinx cavity shunting procedures. Journal of Neurosurgery. 1998; 89: 382-388.

15. Hida K, Iwasaki Y, Koyanagi I, Sawamura Y, Abe H. Surgical indication and results of foramen magnum decompression versus syringosubarachnoid shunting for syringomyelia associated with Chiari I malformation. Neurosurgery. 1995; 37: 673-678.

16. Klekamp J, Samii M. Syringomyelia: diagnosis and management. 2002; Springer: Berlin Heidelberg New York Tokyo: p 195. 